\title{
Pregnancy after excision of a yolk sac tumour of the ovary followed by chemotherapy
}

\section{'SR Ratnapala and ${ }^{2}$ SD Ratnapala}

(Index words: Chemotherapy, histopathology, surgery)

\section{Introduction}

A malignant ovarian tumour was excised and a course of chemotherapy given. Within 20 months of treatment, conception was achieved.

\section{Case report}

A 26-year old woman presented with a 2-day history of severe abdominal pain, distension, breathlessness and fever. Clinical examination revealed a large tender abdominal mass arising from the pelvis.

She was previously treated for subfertility with three consecutive cycles of clomiphene citrate. An ultrasound scan of the abdomen showed a large solid ovarian mass with a few cystic areas. Urine for beta-HCG was negative. The alpha fetoprotein level was over $350000 \mathrm{ng} / \mathrm{mL}$. A chest xray showed small pleural effusions on both sides.

Laparotomy revealed a large friable fungating mass, $15 \times 15 \mathrm{~cm}$, rupturing its capsule at many places, and a moderate amount of ascites. This mass originated from the left ovary. The left fallopian tube could not be visualised as it was entangled in the mass. The entire mass was excised, conserving the right ovary and the tube, which appeared normal. No adhesions were found. A sample of ascitic fluid was sent for cytology. No lymph node involvement was detected. The liver appeared unaffected. A sample of omentum was also sent for histology. The patient's postoperative recovery was uneventful and she was discharged from hospital after 3 days.
The pathologist reported a germ cell tumour, composed of vacuolated network of embryonal cells, hyaline globules and Schillar-Duval bodies. The appearance was that of a yolk sac tumour of the ovary. Omentum was congested and infiltrated with chronic inflammatory cells with no malignant cells present. Ascitic fluid contained inflammatory mesothelial cells. No malignant cells were present.

The patient was subsequently referred to an oncologist, who arranged for a CT scan of the whole abdomen. This showed normal abdominal viscera and the remaining right ovary showed a cyst within. Alpha fetoprotein following surgery had declined from $350000 \mathrm{ng} / \mathrm{mL}$ to $5 \mathrm{ng} / \mathrm{mL}$.

After confirming the diagnosis, a course of chemotherapy consisting of five cycles of intravenous infusions of cisplatin, etoposide and bleomycin was instituted. The patient tolerated chemotherapy well. Serum alpha fetoprotein was further monitored. A subsequent ultrasound scan reported a normal uterus and the right ovary $20 \times 28$ x $38 \mathrm{~mm}$ with multiple small follicles. She was discharged from the hospital and told to expect normal menses in a few months. Regular menstrual cycles resumed 6 months after chemotherapy. She was advised to take $5 \mathrm{mg}$ of folic acid daily, anticipating conception.

She conceived 20 months after chemotherapy and delivered by caesarean section a healthy baby girl near term.

${ }^{1}$ Conslutant in Obstetrics and Gynaecology, and 2 Senior House Office (Surgical), Apollo Hospital, Colombo. Correspondence: SRR, 231, Kirula Road, Colombo 5 (Competing interests: none declared). Received 24 May 2004 and revised version accepted 28 September 2004. 


\section{Discussion}

Germ cell tumours may occur in both gonadal and extra-gonadal locations. Locations and specific tumour types depend on the age. The tumours are grouped together because they all appear to arise from post-meiotic germ cells. Most of the malignant tumours produce markers that can be assessed serologically [1].

Several theories exist about the origin of these tumours. The best evidence suggests that most are due to abnormal differentiation of fetal germ cells that arise from the fetal yolk sac. Normal migration of these germ cells may cause gonadal tumours. These tumours are found in the midline or gonads. Frequencies of the most common sites are sacro-coccygeal $40 \%$, ovary $25 \%$, testicle $12 \%$, brain $5 \%$, and the other $18 \%$ are in the neck and mediastinum [1].

The survival is linked to the degree of immaturity of the teratoma. A close histological evaluation of immature teratomas shows a good correlation between the degree of immaturity and the presence of microscopic foci of frankly malignant elements which are typically yolk sac tumours. They may also represent primitive neuro-ectodermal tumours. The risk of recurrence appears to be related to the degree of immaturity. The likelihood of recurrence depends on the site of the tumour as well as the completeness of the resection. Sacrococcygeal teratomas are more likely to recur than those in the ovary or other sites. Molecular, biological and cytogenic studies are providing a firmer scientific basis to these observations [1].

Before recent chemotherapeutic successes, 10-year survival rate ranged from $25 \%$ for embryonal carcinoma to $75 \%$ for dysgerminomas. Today overall survival rate is greater than $80 \%$. Platinum-based chemotherapy is the generally preferred approach for initial treatment following an initial surgical procedure [2,3].The standard surgical approach to advanced dysgerminoma is complete resection, if possible. However, given the effectiveness of platinum-based chemotherapy of these tumours, extensive mutilating surgery that sacrifices vital structures is not justified. Studies in adult women have demonstrated that the survival outcome is excellent and fertility can be preserved by unilateral salpingo-oopherectomy followed by platinum-based chemotherapy. The chemotherapy combinations comprising cisplatin, etoposide and bleomycin are now preferred because of a low relapse rate and short treatment period [2, 3].

Until now, only a few studies have evaluated the reproductive outcome of patients treated with this protocol. Furthermore, there are no data regarding the effect of chemotherapy combinations commonly used on the physical and neurological development of children born to women previously exposed to these agents [4].

Due to the rare nature of these tumours it is difficult to study a large number of patients. In a study over a period of 20 years, two major changes have occurred since the 1970s that have favourably affected the outcome, namely the shift from radiation therapy to chemotherapybased treatment and the change from cyclophosphamidebased chemotherapy to platinum-based chemotherapy.

To date, children born to these patients have not experienced an increased risk of congenital malformations. Patients with this disease and their family members should be reassured about the high chance of retaining the patient's ability to conceive and have normal children.

\section{References}

1. Bakri YN, Ezzat A, Akhtar, Dohami, Zahrani. Malignant germ cell tumors of the ovary. Pregnancy consideration. European Journal of Obstetrics and Gynecology and Reproductive Biology 2000; 90: 87-91.

2. Gershanson DM, Morris M, Cangis A.Treatment of malignant germ cell tumor of the ovary with bleomycin, etoposide and cisplatin; Clinical Oncology 1990; 8: 715-20.

3. William S, Blessing JA, Lucin SY. Adjuvant therapy of ovarian germ cell tumor with isplatin, etoposide and bleomycin. A trial of the gynecologic oncology group. Clinical Oncology 1994; 12: 701-6.

4. Jacob T, Zelterman D. Reproductive function after conservative surgery and chemotherapy for malignant germ cell tumor of the ovary. Obstetrics and Gynaecology 2003; 101: 251-7. 